\title{
Use of zebrafish (Danio rerio) as a model for research in toxicological studies
}

\author{
Kalpana Singh* \\ Department of Zoology, D.N. (P.G.) College, Meerut 250004 (Uttar Pradesh), India \\ Satyendra Kumar Kashyap \\ Department of Zoology, D.N. (P.G.) College, Meerut 250004 (Uttar Pradesh), India \\ Vandana Garg \\ Department of Zoology, D.N. (P.G.) College, Meerut 250004 (Uttar Pradesh), India
}

*Corresponding author. Email: ksingh0696@gmail.com

\section{Article Info}

https://doi.org/10.31018/

jans.v13i3.2767

Received: June 8, 2021

Revised: July 30, 2021

Accepted: August 4, 2021

\section{How to Cite}

Singh, K. et al. (2021). Use of zebrafish (Danio rerio) as a model for research in toxicological studies. Journal of Applied and Natural Science, 13(3), 846 - 852. https://doi.org/10.31018/jans.v13i3.2767

\begin{abstract}
Danio rerio, commonly known as zebrafish, is a freshwater aquarium fish and is native to parts of South Asia. It is considered an important organism for analyzing the noxious effects of toxicants and pollutants of the environment. In terms of the molecular signaling pathway, molecular properties, organ functions and structures, and neurogenesis, zebrafish are similar to certain other higher-order vertebrates. The 3Rs, refinement,reduction, and replacement in researchhavegradually evolved with time. The accumulation of toxicants in the environment and the human health conditions from exposure to toxicants present in the environment is a serious concern, and zebrafish serves as an excellent model to research such effects. The three Rs are met by zebrafish, larvae can also be used to discover harmful medication compounds, permitting safer compounds to be explored in model organisms and it could also be used to substitute certain toxicological testing.Also, because embryos are fertilized outside and are visible during the initial days of life, the early larval model of zebrafish enables flexibility to animal research study, subsequently reducing the number of animals employed in experiments.For various experimentation studies, the larva of the zebrafish is proved to be a useful model for the system. Thus, being a good test system, zebrafish are used in environmental health and safety studies. This review focuses on the toxicological studiesin zebrafish and outlines the toxicological studies done on zebrafish with arsenic and 2,3,7,8-Tetrachlorodibenzo-p-dioxin (TCDD) as well as microplastic toxicity.
\end{abstract}

Keywords: Zebrafish, Arsenic, 2,3,7,8-Tetrachlorodibenzo-p-dioxin, Microplastics

\section{INTRODUCTION}

Toxic substances directly affect all kinds of fish by negatively influencing the lifespan, reproductive system, and growth of the embryo, morphology, and neural behaviour. Endocrine-disrupting chemicals (EDCs) are often used in food packaging, fine particulate matter, household goods, and personal care products, as well as naturally occurring substances like metals (Bambino and Chu, 2017). Environmental chemicals such as asbestos, glyphosphate, Per- and Polyfluoroalkyl substances (PFAS), Polychlorinated biphenyls etc.,are linked with both acute and long-term toxicity in humans (Landriganet al., 2016). Innumerable contaminants and toxins such as mercury, arsenic, chlorinated naphthalenes, dioxins and furans, etc., are released daily in the environment from various sources. Dangers are posed by the growing list of synthetic contaminants and natu- rally occurring chemicals such as metals, which may also affect the health of not only human beings but also domestic animals(Tong et al., 1996; Bellinger, 2016).Microplastics are new, poorly known pollutants that are causing worldwide awareness. To describe microplastics toxicity and link it to the cellular level of response, it is critical to know the molecular interactions between organisms and microplastics. Furthermore, the environmental risk created can be tracked and counter-measures can be established according to the need (Umamaheswari et al., 2020). These are a type of new pollutant that has gotten a lot of coverage from the social and scientific communities in recent decades. These are typically characterized as plastic particles smaller than $5 \mathrm{~mm}$ that are formed due to the widespread use of plastic products. According to the latest simulation result, worldwide plastic trash will triple between 2015 and 2060, hitting around 2.7 billion 
tonnes (Zhao et al., 2021).

The zebrafish (Danio rerio) belong to the order Cypriniformes and family Cyprinidae (minnow family), was first described in 1822 by Hamilton from the river Kosi, Bengal, India (Arunachalam et al., 2013). It is a very popular aquarium fish and is native to the freshwater and rice fields of South Asia i.e., it is found in the regions of Bhutan, Pakistan, Nepal, India, Burma and Bangladesh. The South Himalayas form the northern boundary, stretching from the Sutlej River Basin on the Pakistan-India border to Arunachal Pradesh in northeast India. Zebrafish have been introduced to the various region of the United States such as New Mexico, Connecticut, California, and Florida, and elsewhere to Colombia and Malaysia (Engeszer et al., 2007). Zebrafish is found to be an ideal organism for toxicological studies of drug compounds, heavy metals, industrial waste products, pesticides, and agrochemicals. It is also accustomed to study human diseases through genetic alterations, studies of the liver, heart, kidney, immune, hematopoietic, and other systems. Genetic alterations can be easily measured in zebrafish and its embryo's growth allows easy monitoring for the identification of fluorescently labeled reporters and proteins. The genetic alteration in zebrafish was first reported in 1981. Mostly nano-sized microplastics can normally enter the developing chorion and concentrate inside the developing embryo (Cheng et al., 2021). During the embryonic stage of the zebrafish, the membrane of the embryo works as a barrier, inhibiting microplastics and keeping such particles outside the membrane. These microplastics can get into the body of zebrafish through the oesophagus once the embryonic membrane breaks. According to a recent report, microplastics were discovered in the chorion amniotic, foetal, and maternal membranes of the human placenta for the first time (Ragusa et al., 2021). Zebrafish at an early embryonic stage is considered a good model organism for assessing the consequences of the microplastics as the embryo is surrounded by a covering of egg cell membrane, which could replicate the protective barrier of the embryo (Zhao et al., 2021). Chorion of developing embryo zebrafish is a unique biological feature that protects the developing embryo till the zebrafish's larvae emerge (Cheng et al., 2021). Reproductive toxicity, locomotor and neurological toxicity, developmental toxicity, genotoxicity, immunotoxicity, oxidative stress, and intestinal damage and metabolome are amongst the key outcomes in studies conducted on zebra fish employing microplastics either alone or in amalgam with other compounds(Bhagat et al., 2020). In terms of toxicological science, genetic manipulation can establish disease models, pace screening assays by evaluating fluorescent reporters, and provide simple tools to study the various processes involved. In this review, we have outlined various toxicological studies that will help understand zebrafish's processes in safety evaluation studies. The zebrafish model is also accustomed to study the probable relationship between exposures to toxicants and their effect on a vertebrate.

\section{HISTORICAL PERSPECTIVE}

The Toxic Substances Control Act was passed by the United States Congress in 1976, giving the Environmental Protection Agency the power to mandate screening and documenting and impose limits on the production and safe use of chemicals and their combinations (https://www.epa.gov/laws-regulations/summar y-toxic-substances-control-act). The Department of Health, Education, and Welfare, of the United States, now known as the Department of Health and Human Services, developed the National Toxicology Program in 1978at United States as an intergovernmental system to address legislative, public, and scientific concerns that chemical emissions are related to human diseases and disabilities (Xie et al., 2017).

Streisinger et al. (1981) suggested a vertebrate model, i.e., zebrafish for the first time, to investigate the alterations that occurred from environmental carcinogens.Carvan et al. (2000)reported thatthe zebrafish's embryo and larvae are ideal for biomonitoring, and transgenic animals. This has enabled the creation of transgenic lines that trigger a fluorescent protein or other observable readouts in the existence of pollutants, providing a framework to evaluate the amount of response and tissue sensitivity of the response. From fertilization to larval stages, toxins can simply apply to the embryo, and the emerging and visible stages of the zebrafish can be examined for mortality and developmental malformations. While juvenile phase and adult zebrafish are not see-through, the unpigmented caspermutant line can be crossed to genetically modify fluorescent reporters to help detect and visualize organ systems in adult zebrafish (White et al., 2008).A consortium of International pharmaceutical companies in 2009 designed a zebrafish production assay that could accurately describe a range of teratogenic and nonteratogenic substances. When the results of such toxicological studies were contrasted to mammalian statistics, it was discovered that there was about 60 to 70 percent approximate conformity (Gustafson et al., 2012). For a testing range of 31 chemicals, developmental toxicity analysis was designed of the dechorionated embryo of zebrafish to determine the level of adverse effects and the dosage that causes $25 \%$ mortality $\left(\mathrm{LC}_{25}\right)$ (Brannen et al., 2010). The wide spectrum of cytochrome P450 (Cyp) genes necessary for xenobiotic processing and detoxification are expressed in zebrafish (Goldstone et al., 2010). Forthe last three decades, to classify teratogens and discover common toxicants' mechanisms, zebrafish have been considered an ideal model 
(Gamse and Gorelick, 2016). Bambino and Chu, (2017) reported the capability to investigate molecular pathways of developmental and health impacts in a living vertebrate population and early detection of compound toxicity on zebrafish. Some cypgenes were detected by Saad et al. (2016)in the genome assembly (GRCz10) of the zebrafish. Using zebrafish is a medium to provide useful knowledge about the short- and long-term health impacts of toxicant exposure on humans in toxicology studies (Gamseand Gorelick, 2016).

\section{ZEBRAFISH AS AN IDEAL MODEL}

Zebrafish are an excellent alternative to mammals for toxicological experimentation because of their breeding, high fecundity, rapid growth, small size, and seethrough young ones. Certain organs of the zebrafish play the same role as their human counterparts and have well-preserved anatomy. Several zebrafish of about only $4 \mathrm{~cm}$ in length can be comfortably placed in a regular aquarium, and industrial services for automatic monitoring the quality control of water.

A single pair's successful breeding produces several fertilized embryos outside the body that develop efficiently. Most of the organs are nearly finished in the gastrointestinal tract during early growth, within 48- and 72-hours post-fertilization. The gut, pancreas, and liver are developed properly around 76 hours postfertilization, and the gastrointestinal tract is fully developed by 96 hours post-fertilization. The development of young zebrafish is easily visible. Moreover, zebrafish are used to investigate the effects in the physiology and morphology due to toxicants. The genome sequencing of zebrafish has been done, and in contrast to the human genome, it was discovered that 70 percent of the total human genes had a homolog to zebrafish, and 82 percent of human genes linked to the disease had a homolog to zebrafish (Cassar et al., 2020).For a long time, Zebrafish has been identified as a study model in the fields of genetics, molecular biology, developmental biology, and ecological toxicology. The majority of toxicology research done on zebrafish has focused on environmental pollutants. In zebrafish, independent feeding starts about fivedays after fertilization, as a result, working with embryos/larvae of zebrafish under the age of $5 \mathrm{dpf}$ (days post-fertilization) and independent feeding, yolk consumption, and swimming behavior appear at 120 hours post-fertilization may be viewed as a substitute for animal toxicity experimentation (Strahle et al., 2012).

\section{MECHANISMS OF TOXICITY}

Zebrafish are used to study the various mechanisms of toxic compounds and chemicals and diseases associated with them and assess clinical candidates or preven- tive measures to reduce the impact of environmental pollutants to translate these findings to various diseases. This review paper illustrates some of the mechanistic insights gained from zebrafish exposure, such as inorganic arsenic (As), 2,3,7,8-Tetrachlorodibenzo-pdioxin (TCDD), and microplastic toxicity.

\section{Arsenic toxicity}

Arsenic (As) is a natural crystalline metalloid. It is the 20th most copious element found in the crust of the earth and found in the plentiful amount in the human, ranking 12th. It is an extremely toxic environmental pollutant that poses a critical threat to the environment and affects the health of humans. Chronic exposure to As has been associated with a higher risk of many disorders and cancers in humans, including pathogenic enlargement of the liver, fatty degeneration, skin, bladder, kidney cancer, liver cirrhosis, etc. (Wang et al., 2008; Kumar et al., 2019). While it is amongst the most frequent toxic metalloid pollutants in potable water and can also be found in significant concentrations in food products like apple juice and rice (Davis et al., 2012; Sauve, 2014), the accurate process of its toxicity is still not in knowledge.

The trivalent arsenic-specific methyltransferase (ZAs3Mt) and aquaglyceroporins are enzymes necessary for the metabolism and uptake of inorganic As, respectively, in zebrafish. Early research on inorganic As exposure in zebrafish provides an explanatory evaluation of the effects caused on morphological growth of larvae and embryos, revealing cardiovascular malformations and acute toxicity (Seok et al., 2007; Li et al., 2009). The rate of As metabolism and the development of its unique metabolites by the zebrafish is still unknown.Some studies of zebrafish embryos reported the reduced expression of Dvr1a factor important in mesoderm development and the establishment of left-right asymmetry. Folic acid inhibited arsenic-induced toxicity in zebrafish by reducing the development of ROS (reactive oxygen species) is also reported.In the liver of an adult, to analyze the molecular processes of toxicity, metabolomic and transcriptomic strategies were also studied (Lam et al., 2006; Yang et al., 2007; Xu et al., 2013; Li et al., 2016). A group of biomarker genes for predicting As toxicity was detected using gene ontology and mechanical assessment of RNA-SAGE results (Xu et al., 2013). Four core parameters: jun, r2f2, apoE, and $k-r$ as, were up-regulated in the liver following exposure, according to network analysis. These factors have been linked with the development of As-induced liver problems: $\mathrm{Nr} 2 \mathrm{f} 2$ controls a number of genes involved in drug metabolism and oxidative stress, while $\mathrm{k}$ -ras and jun are well-known oncoproteins, and ApoE is needed for lipoprotein production.

Short-term exposure of lower concentrations in the skin, eyes, and liver of six-month-old fish, causes rapid 
heartbeat and neuro-developmental disorders during larval stages. At about 90 days of age, the offspring of exposed fish had lower biomass than their siblings which were unexposed (Hallauer et al., 2016). Gas chromatography and mass spectroscopy (MS) were used to examine metabolic alterations in the liver of adults after acute exposure, detecting 34 probable metabolite markers. Furthermore, though microanatomy explorations of the liver of exposed zebrafish revealed pathological variations and lipid droplet deposition, liver function tests revealed limited modifications ( $\mathrm{Li}$ et al., 2016), indicating that metabolic changes could be fragile diagnose As-induced variation in the functioning of the liver. Even though the zebrafish is used to investigate toxicity caused by Asis relatively new, this model has provided valuable cognizance into both the shortterm (Xu et al., 2013; Li et al., 2016) and long-term effects of Arsenic toxicity. Kabir et al. (2020) treated zebrafish with $2 \mathrm{mM}$ of arsenic and compared the results with the untreated zebrafish. He observed arsenic -treated embryos show a unique phenotype three days after fertilization, which includes increased egg mass, aberrant heart rhythm, dechorionated embryos, pericardial edema, and improper head formation. Therefore, the researchers concluded that treating embryos with arsenic can lead to aberrant development. Ancestral generation of zebrafish was treated with $500 \mathrm{ppb}$ of arsenic for 150 days. When ancestral generation was raised, they produced intergeneration i.e. F1 generation and trans generation i.e., F2 generation. Through this study, researchers evaluated the cognitive and motor behavior, epigenetic markers, and neurodevelopmentrelated genes, on the ancestral generation and transgeneration. Treating ancestral generation with arsenic during development affects motor behaviour and enhanced anxiety-like behaviours, which were passed down to the transgeneration. Additionally, between the ancestral generation and transgeneration generations, there was a decrease in neurotrophic factor expression derived from the brain and in the nervous system, rise in methylation on histone H3K4me3 (Valles et al., 2020).

\section{TCDD toxicity}

In Zebrafish, TCDD (2,3,7,8-Tetrachlorodibenzo-pdioxin) is amongst the most broadly studied environmental pollutant (Carney et al., 2006). A polychlorinated dibenzo-p-dioxin, a constant anthropogenic lipophilic environmental pollutant is widely contained in the atmosphere due to solid waste combustion and industrial output. Human industrial and environmental pollution has been connected with various diseases, comprising cardiovascular disease, cancer, endometriosis, cardiovascular disease, thyroid hormone deficiency, diabetes, and testosterone deficiency. TCDD-induced endocrine dysfunction and reproductive toxicity, skeletal deformi- ties, and cardiovascular toxicity have also been studied using zebrafish. Deficiency of Ahr2 prevents developmental deformities in zebrafish larvae and embryos subjected to TCDD. The Cyp1a genetically modified reporter zebrafish were used to analyze the processes of toxicity by TCDD and to recognize effects of TCDD target organs (Mattingly et al., 2001; Kim et al., 2013; $\mathrm{Xu}$ et al., 2015); although, mechanisms other than Cyp1a1 lead to TCDD's developmental toxicity, as zebrafish cyp1a inactivation does not avoid TCDDinduced mutations.

Ovarian activity and follicle development have also been appeared to be affected by TCDD (Heidenet al., 2008; Baker et al., 2014). TCDD has been connected with multiple disabilities in the female reproductive system in a variety of fish species. Genes associated with follicle development and estradiol formation and the genes that encode structural proteins Lgals3I and Krt4 were all down-regulated after treatment to TCDD.

Amongst the most noticeable effects of TCDD toxicity in zebrafish is cardiovascular toxicity. Analyzing gene expression over time was done to learn about the biological processes that are disrupted in response to TCDD treatment. In zebrafish, treated with TCDD, a "cell cycle gene cluster" was inhibited, whereas negative regulators of cell cycle advancement were up-regulated, implying that TCDD-induced cardiovascular toxicity is caused by a reduction in cardiomyocyte amount (Carney et al., 2006). The inability of TCDD-induced, development of the heart valve in the zebrafish was appeared to be affected by elevated and ectopic transcription of Notch1b and Bmp4 transcripts in the region of embryonic cardiac valve development (Mehta et al., 2008).

Another commonly diagnosed defect linked to TCDD treatment is skeletal deformity. The Ahr2/Arnt1 signaling pathway is needed for cranial deformities in TCDDtreated zebrafish (Praschet al., 2003; 2006). Adult fish with scoliosis have been seen after being exposed to TCDD during developmental or larval stages.

\section{Microplastics toxicity}

Research on the toxicity of microplastics along with other compounds has revealed that these induce oxidative stress by generating reactive oxygen species (ROS), inducing degradation of the biological membranes, and causing metabolic changes (Umamaheswari et al., 2020). Umamaheshwari et al. (2020) treated zebrafish with polystyrene microplastics and observed adsorption of microplastics in the gills and gastrointestinal tract during eating and breathing, which caused restriction in oxygen absorption, oxidative damage, digestive process, energy deprivation, and mortality. Treatment with polystyrene microplastics resulted in gene regulated physiological and biochemical effects at different concentration levels. Zhao 
et al. (2021) discovered that after being exposed to polyethylene microplastics, the makeup of the microbiota in the larval stage of the zebrafish changed considerably. Malafaiaet al. (2020) after exposing the embryo of zebrafish to microplastics, observed premature birth in contrast to the unexposed group. The early hatching in the exposed media did not survive longer, resulting in a higher percentage of larval mortality. Microplastics caused various behavioural and anatomical abnormalities in larvae, including tail bending upward or downward (vascular abnormality), pericardial edema, jaw abnormalities, reduced heart rate, string heart, and swimming ability. Insights into the molecular basis of microplastic toxicity have been provided by changes in metabolites reported by amino acids and fatty acids (Bhagat et al., 2020). It has been found that long-term treatment to polystyrene microplastic in the water can contaminate the water, harming the sexual organs of the zebrafish. This research highlighted polystyrene microplastic treatment as a significant indicator of reproductive stress in zebrafish, and it implies that prolonged exposure may affect fertility further(Qianget al., 2021).

From the above information, this can be inferred that zebrafish is an ideal organism to be studied in terms of toxicity and shows various changes upon exposure to various pollutants. Exposing zebrafish to different concentrations of arsenic shows that arsenic affects the growth of zebrafish in the initial stages of development, causing cardiovascular deformities. When a low concentration of arsenic is given to the zebrafish for a short time, it causes neurodevelopmental disorders and increases the heartbeat rate. By performing microanatomy, variations in the pathology reserves and deposition of liquid droplets have been seen. Research on the effects caused by As toxicity in zebrafish provides insight into the processes underlying these health consequences and enables for more easily examining intergenerational consequences. Understanding the mechanisms and processes of As toxicity in zebrafish will help researchers to understand as to how As affects human health.

TCDD is one of the most studied pollutants occurring in the environment. When TCDD is given to zebrafish, it shows cardiovascular toxicity as the most noticeable effect, dysfunction of the endocrine system, activity of ovaries, and follicle development was also affected, indicating dysfunctioning of the reproductive system in females and most commonly seen malformation upon exposure was of skeletal deformity. TCDD isperhaps the most researched environmental pollutant. Zebrafish studies about the processes and molecular mechanisms causing TCDD toxicity have revealed humanrelevant effects, including cardiovascular and reproductive disorders

Research on microplastics along with different com- pounds has shown various results in zebrafish. When zebrafish were exposed to polystyrene microplastics, they showed limited absorption of oxygen, oxidative damage, listless and mortality. Premature birth of the zebrafish was seen when exposed to polyethylene microplastics and the early hatched progeny died early. Exposure to microplastics shows abnormalities in behaviour and anatomy of larvae. The amount of reactive oxygen species (ROS) in reproductive organs might be a useful early predictor of damage to the reproductive system caused by exposure to microplastics. Environmental levels of microplastics in water bodies are projected to rise more in the future. Moreover, regular variations in concentration might result in larger surges, putting the actual exposure of aquatic life in jeopardy

\section{Conclusion}

The zebrafish is a versatile model that falls under the category of an ideal organism to study the toxicity caused by various toxicants. In initial screening assays, zebrafish can also be used to assess the new pharmaceutical drugs toxicity in a high-throughput manner. The scalability, repeatabilityand outcome connected with xenobiotic screens will benefit from the advancement of technologies which allows the evaluation of hundreds of metabolites, genes, and proteins. The fundamental advantage of utilizing zebrafish in toxicology and environmental, medical studies is that their amalgamation of developmental traits results in a system with the advantages of both in vivo and in vitro models. Though larval behavioural assays are currently well mechanized, increasing the use and standardization of adult behavioural analyses will produce high-quality data and illustrate functional outcomes of xenobiotic treatment. As a result, zebrafish investigations have revealed crucial information about the effects of environmental pollutants on developing a vertebrate system. These findings can aid in selecting safer drugs for mammalian research, the discovery of toxicity processes, and the identification of therapies that may reduce the toxicity of potential medicines.

\section{Conflict of interest}

The authors declare that they have no conflict of interest.

\section{REFERENCES}

1. Arunachalam, M., Raja, M., Vijayakumar, C., Malaiammal, P., \& Mayden, R. L. (2013). Natural history of zebrafish (Danio rerio) in India. Zebrafish, 10(1). DOI: 10.1089/zeb. 2012.0803

2. Baker, T. R., King-Heiden, T. C., Peterson, R. E. \& Heideman, W. (2014). Dioxin induction of transgenerational 
inheritance of disease in zebrafish. Mol Cell Endocrinol, 398(1-2), 36-41. https://doi.org/10.1016/j.mce.2014.08.01 1

3. Baker, T. R., Peterson, R. E. \&Heideman, W.(2014). Using zebrafish as a model system for studying the transgenerational effects of dioxin. Toxicol. Sci., 138(2), 403-411. DOI: 10.1093/toxsci/kfu006

4. Bambino, K. \& Chu, J.(2017). Zebrafish in toxicology and environmental health. Curr. Top. Dev. Biol., 124, 331-367. DOI: 10.1016/bs.ctdb.2016.10.007

5. Bellinger, D. C. (2016). Lead contamination in flint--An abject failure to protect public health. N. Engl. J. Med., 374(12), 1101-1103. DOI: 10.1056/NEJMp1601013

6. Bhagat, J., Zang, L., Nishimura, N. \& Shimada, Y. (2020). Zebrafish: An emerging model to study microplastic and nano plastic toxicity. Science of The Total Environment, 728.DOI: 10.1016/j.scitotenv.2020.138707

7. Brannen, K. C., Panzica-Kelly, J. M., Danberry, T. L. \& Augustine-Rauch, K. A.(2010). Development of a zebrafish embryo teratogenicity assay and quantitative prediction model. Birth Defects Res. B Dev. Reprod. Toxicol., 89(1), 66-77. DOI: 10.1002/bdrb.20223

8. Carney, S. A., Chen, J., Burns, C. G., Xiong, K. M., Peterson, R. E. \& Heideman, W.(2006). Aryl hydrocarbon receptor activation produces heart-specific transcriptional and toxic responses in developing zebrafish. Mol Pharmacol. 70(2), 549-561.https://doi.org/10.1002/bdrb.20223

9. Carney, S. A., Prasch, A. L., Heideman, W. \& Peterson, R. E. (2006). Understanding dioxin developmental toxicity using the zebrafish model. Birth Defects Res. A Clin. Mol. Teratol., 76(1), 7-18. DOI: 10.1002/bdra.20216

10. Carvan, M. J. 3rd, Dalton, T. P., Stuart, G. W. \&Nebert, D. W. (2000). Transgenic zebrafish as sentinels for aquatic pollution. Ann. N. Y. Acad. Sci., 919, 133-147.DOI: 10.111 1/j.1749-6632.2000.tb06875.x

11. Cassar, S.,Adatto, I., Freeman, J. L., Gamse, J. T., Iturria, I., Lawrence, C., Muriana, A., Peterson, R. T., Cruchten, V. S. \& Zon, L. I.(2020). Use of zebrafish in drug discovery toxicology. Chem. Res. Toxicol., 33(1), 95-118.https:// doi.org/10.1021/acs.chemrestox.9b00335

12. Cheng, H., Feng, Y., Duan, Z., Duan, X., Zhao, S., Wang, Y., Gong, Z. \& Wang, L. (2021). Toxicities of microplastic fibers and granules on the development of zebrafish embryos and their combined effects with cadmium. Chemosphere, 269. https://doi.org/10.1016/j.chemosphere.202 0.128677

13. Davis, M. A., Mackenzie, T. A., Cottingham, K. L., GilbertDiamond, D., Punshon, T. \& Karagas, M. R. (2012). Rice consumption and urinary arsenic concentrations in U.S. children. Environ. Health Perspect., 120(10), 1418-1424. DOI: 10.1289/ehp.1205014

14. Engeszer, R. E., Patterson, L. B., Rao, A. A. \& Parichy, K. D.M. (2007). Zebrafish in the wild: A review of natural history and new notes from the field. Zebrafish, 4 (1), 2139. doi:10.1089/zeb.2006.9997.

15. Gamse, J. T. \& Gorelick, D. A. (2016). Mixtures, metabolites, and mechanisms: Understanding toxicology using zebrafish. Zebrafish, 13(5), 377-378. DOI: 10.1089/ zeb.2016.1370

16. Goldstone, J. V., McArthur, A. G., Kubota, A., Zanette, J., Parente, T., Jönsson, M. E., Nelson, D. R. \& Stegeman, J. $J .(2010)$. Identification and developmental expression of the full complement of Cytochrome P450 genes in
Zebrafish. BMC Genomics, 11, 643.

17. Gustafson, A. L., Stedman, D. B., Ball, J., Hillegass, J. M., Flood, A., Zhang, C. X., Panzica-Kelly, J., Cao, J., Coburn, A., Enright, B. P., Tornesi, M. B., Hetheridge, M., \& Augustine-Rauch, K. A. (2012). Inter-laboratory assessment of a harmonized zebrafish developmental toxicology assay - progress report on phase I. Reproductive toxicology (Elmsford, N.Y.), 33(2), 155-164. https:// doi.org/10.1016/j.reprotox.2011.12.004

18. Hallauer, J.,Geng, X., Yang, H. C., Shen, J., Tsai, K. J. \& Liu, Z. (2016). The effect of chronic arsenic exposure in zebrafish. Zebrafish, 13(5), 405-412. doi: 10.1089/zeb.20 16.1252

19. Heiden, T. C., Struble, C. A., Rise, M. L., Hessner, M. J., Hutz, R. J. \& Carvan, M. J. 3rd.(2008). Molecular targets of 2,3,7,8-tetrachlorodibenzo-p-dioxin (TCDD) within the zebrafish ovary: insights into TCDD-induced endocrine disruption and reproductive toxicity. Reprod. Toxicol., 25 (1), 47-57.doi: 10.1016/j.reprotox.2007.07.013

20. Kabir, T., Anwara S., Mourosia J. T., Hossain, J., Rabbaneb, M. G., Rahmand, M. M., Tahsina, T., Hasane, M. N., Shillf, M. C. \& Hosen, M. J. (2020). Arsenic hampered embryonic development: An in vivo study using local Bangladeshi Danio rerio model. Toxicology Reports, 7, 155-161.DOI: 10.1016/j.toxrep.2019.12.009

21. Kim, K. H., Park, H. J., Kim, J. H., Kim, S., Williams, D. R., Kim, M. K., Jung, Y. D., Teraoka, H., Park, H. C., Choy, H. E., Shin, B. A. \& Choi, S. Y.(2013). Cyp1a reporter zebrafish reveals target tissues for dioxin. Aquat.Toxicol., 134-135, 57-65.DOI: 10.1016/j.aquatox.2013.03.010

22. Kumar, V., Vogelsang, L., Seidel, T., Schmidt, R., Weber, M., Reichelt, M., Meyer, A., Clemens, S., Sharma, S. S. \& Dietz, K. J.(2019). Plant Cell Environ., 42(2), 574590. https://doi.org/10.1111/pce.13441

23. Lam, S. H., Winata, C. L., Tong, Y., Korzh, S., Lim, W. S., Korzh, V., Spitsbergen, J., Mathavan, S., Miller, L. D., Liu, E. T. \& Gong, Z.(2006). Transcriptome kinetics of arsenicinduced adaptive response in zebrafish liver. Physiol Genomics, 27(3), 351-361. DOI: 10.1152/physiolgenomics.00 201.2005

24. Landrigan, P. J.(2016). Children's environmental health: A brief history. Acad. Pediatr., 16(1), 1-9. DOI: 10.1016/j.ac ap.2015.10.002

25. Li, C., Li, P., Tan, Y. M., Lam, S. H., Chan, E. C. \& Gong, Z. (2016). Metabolomic characterizations of liver injury caused by acute arsenic toxicity in zebrafish. PLoS One, 11(3). https://doi.org/10.1371/journal.pone.0151225

26. Li, D., Lu, C., Wang, J., Hu, W., Cao, Z., Sun, D., Xia, H. \& $\mathrm{Ma}, \mathrm{X} .(2009)$. Developmental mechanisms of arsenite toxicity in zebrafish (Danio rerio) embryos. Aquat. Toxicol., 91(3), 229-237. DOI: 10.1016/j.aquatox.2008.11.007

27. Mattingly, C. J., McLachlan, J. A. \& Toscano, W. A. Jr. (2001). Green fluorescent protein (GFP) as a marker of aryl hydrocarbon receptor (AhR) function in developing zebrfish (Danio rerio). Environ. Health Perspect., 109(8), 845-849.DOI: 10.1289/ehp.01109845

28. Malafaia, G., de Souza, A. M., Pereira, A. C.Gonçalves, S., da Costa Araújo, A. P., Ribeiro, R. X. \& Rocha, T. L. (2020). Developmental toxicity in zebrafish exposed to pristine polyethylene microplastics under static and semistatic systems. Science of The Total Environment, 134867.DOI: 10.1016/j.scitotenv.2019.134867 
29. Mehta, V., Peterson, R. E. \&Heideman, W. (2008). 2,3,7,8 -Tetrachlorodibenzo-p-dioxin exposure prevents cardiac valve formation in developing zebrafish. Toxicol. Sci., 104 (2), 303-311. DOI: 10.1093/toxsci/kfn095

30. Prasch, A. L., Tanguay, R. L., Mehta, V., Heideman, W. \& Peterson, R. E. (2006). Identification of zebrafish ARNT1 homologs: 2,3,7,8-tetrachlorodibenzo-p-dioxin toxicity in the developing zebrafish requires ARNT1. Mol Pharmacol.69(3), 776-787.DOI: 10.1124/mol.105.016873

31. Prasch, A. L., Teraoka, H., Carney, S. A., Dong, W., Hiraga, T., Stegeman, J. J., Heideman, W. \& Peterson, R. E. (2003). Aryl hydrocarbon receptor 2 mediates 2,3,7,8tetrachlorodibenzo-p-dioxin developmental toxicity in zebrafish. Toxicol. Sci., 76(1), 138-150. DOI: 10.1093/tox sci/kfg202

32. Qiang, L. \& Cheng, J. (2021). Exposure to polystyrene microplastics impairs gonads of zebrafish (Danio rerio). Chemosphere, 263, 128161.https://doi.org/10.1016/j.che mosphere.2020.128161

33. Ragusa, A., Svelato, A., Santacroce, C. Catalano, P., Notarstefano, V., Carnevali, O., Papa, F., Rongioletti, M. C. A., Baiocco, F., Draghi, S., D'Amore, E., Rinaldo, D., Matta, M. \&Giorgini E. (2021). Plasticenta: First evidence of microplastics in human placenta. Environment International., 146.

34. Saad, M., Cavanaugh, K., Verbueken, E., Pype, C., Casteleyn, C., Van Ginneken, C. \& Van Cruchten, S. (2016). Xenobiotic metabolism in the zebrafish: a review of the spatiotemporal distribution, modulation and activity of Cytochrome P450 families 1 to 3. J. Toxicol. Sci., 41(1), 1-11.DOI: $10.2131 /$ jts. 41.1

35. Sauve, S.(2014). Time to revisit arsenic regulations: comparing drinking water and rice. BMC Public Health, 14 (465)

36. Seok, S. H., Baek, M. W., Lee, H. Y., Kim, D. J., Na, Y. R., Noh, K. J., Park, S. H., Lee, H. K., Lee, B. H., Ryu, D. Y. \& Park, J. H.(2007). Quantitative GFP fluorescence as an indicator of arsenite developmental toxicity in mosaic heat shock protein 70 transgenic zebrafish. Toxicol. Appl. Pharmacol., 225(2), 154-161. DOI: 10.1016/j.taap.2007.07.011

37. Streisinger, G., Walker, C., Dower, N., Knauber, D. \& Singer, F. (1981). Production of clones of homozygous diploid zebra fish (Brachy danio rerio). Nature, 291(5813), 293-296.

38. Tong, S., Baghurst, P., McMichael, A., Sawyer, M. \&Mudge, J. (1996). Lifetime exposure to environmental lead and children's intelligence at $11-13$ years: the Port Pirie cohort study. BMJ, 312(7046), 1569-1575.doi: 10.1 136/bmj.312.7046.1569

39. Umamaheswari, S., Priyadarshinee, S., Bhattacharjee, M.,
Kadirvelu, K. \& Ramesh, M. (2020). Exposure to polystyrene microplastics induced gene modulated biological responses in zebrafish (Danio rerio). Chemosphere, 10.doi: 10.1016/j.chemosphere.2020.128592

40. Valles, S., Sanchez, H. G., Dipp, V. R., Gozalez, D. H., Banuelos, T. N. O., Fraga, J. G. \&Bardullas, U. (2020). Exposure to low doses of inorganic arsenic induces transgenerational changes on behavioral and epigenetic markers in zebrafish (Danio rerio). Toxicol. Appl. Pharmacol., 396. https://doi.org/10.1016/j.taap.2020.115002

41. Strahle, U., Scholz, S., Geisler, R., Greiner, P., Hollert, H., Rastegar, S., Schumacher, A., Selderslaghs, I., Weiss, C., Witters, H. \&Braunbeck, T. (2012). Zebrafish embryos as an alternative to animal experiments--a commentary on the definition of the onset of protected life stages in animal welfare regulations. Reprod. Toxicol., 33(2),128-32. doi: 10.1016/j.reprotox.2011.06.121.

42. Wang, W., Cheng, S. \& Zhang, D. (2014). Association of inorganic arsenic exposure with liver cancer mortality: A meta-analysis. Environ. Res., 135, 120-125.DOI: 10.1016/ j.envres.2014.08.034

43. White, R. M., Sessa, A., Burke, C., Bowman, T., LeBlanc, J., Ceol, C., Bourque, C., Dovey, M., Goessling, W., Burns, C. E.\&Zon, L. I.(2008). Transparent adult zebrafish as a tool for in vivo transplantation analysis. Cell Stem Cell,2(2), 183-189. DOI: 10.1016/j.stem.2007.11.002

44. Xie, Y., Holmgren, S., Andrews, D. M., Wolfe, M. S (2017) Evaluating the impact of the U.S. National Toxicology Program: A case study on hexavalent chromium. Environ Health Perspect., 125(2),181-188. doi: 10.1289/ EHP21

45. Xu, H., Lam, S. H., Shen, Y. \& Gong, Z. (2013). Genomewide identification of molecular pathways and biomarkers in response to arsenic exposure in zebrafish liver. PLoS One, 8(7).https://doi.org/10.1371/journal.pone.0068737

46. Xu, H., Li, C., Li, Y., Ng, G. H., Liu, C., Zhang, X. \& Gong, Z.(2015). Generation of $\mathrm{Tg}$ (cyp1a:gfp) transgenic zebrafish for development of a convenient and sensitive In Vivo Assay for Aryl hydrocarbon receptor activity. Mar Biotechnol (NY), 17(6), 831-840. DOI: 10.1007/s10126015-9669-1

47. Yang, L., Kemadjou, J. R., Zinsmeister, C., Bauer, M., Legradi, J., Müller, F., Pankratz, M., Jäkel, J. \& Strähle, U. (2007). Transcriptional profiling reveals barcode-like toxicogenomic responses in the zebrafish embryo. Genome Biol, 8(10).DOI: 10.1186/gb-2007-8-10-r227

48. Zhao, Y., Qin, Z., Huang, Z., Zhiwei, B.,Luo, T. \&Jin, Y. (2021). Effects of polyethylene microplastics on the microbiome and metabolism in larval zebrafish. Environmental Pollution, 282.https://doi.org/10.1016/j.envpol.2021.1170 39 